\title{
Continuous and Discrete Time Domain Stability Analysis of Composite Weighted Least Norm Solution in Redundancy Resolution
}

\author{
Avik Chatterjee \\ CSIR-CMERI, \\ M.G.Avenue, \\ Durgapur, India.
}

\author{
S.Majumder \\ CSIR-CMERI, \\ M.G.Avenue, \\ Durgapur, India.
}

\author{
I Basak \\ NIT Durgapur, \\ M.G.Avenue, \\ Durgapur, India.
}

\begin{abstract}
Stability aspects of redundancy resolution both in velocity and acceleration level have been investigated for a method which augments the weighted least norm solution by weighted residual of the current joint rate and preferred pose rate in null space. While doing this the first and second order inverse kinematics solutions with redundancy have been re-casted as a feedback control problem, with the classical Close Loop Inverse Kinematics (CLIK) both for range space and null space and its stability conditions are derived for continuous and discrete time domains using Lyapunov and non Lyapunov based stability criteria. The non Lyapunov based analysis is based on the exponential convergence of the task space error system in discrete time domain. For generality the stability conditions of regularized version of CWLS has been analyzed considering the null space contribution which will provide the information of feasible and unfeasible directions that is especially important in near singularity configuration.
\end{abstract}

\section{General Terms}

Inverse Kinematics, Stability.

\section{Keywords}

Null space, range space, stability, redundancy, inverse kinematics, CLIK

\section{INTRODUCTION}

The main difficulty in the study of algorithmic solutions to the inverse kinematics (IK) problem is related to the discrete-time nature of system at one hand, combined with its strong nonlinearity, deriving from the nonlinearity of the kinematics. On the other hand the differential kinematics equation represents a linear mapping between the joint velocity space and the operational velocity space, although it varies with the current configuration. This fact suggests the possibility to utilize the differential kinematics equation to tackle IK problem when it was initially addressed [1]. After wards various researchers resorted to different techniques like classical numerical methods, such as the Newton-Raphson algorithm [2], optimization techniques [3], inverting differential kinematics in a closedloop fashion by viewing the IK problem as a feedback control problem [4], classically knows as Closed Loop Inverse Kinematics (CLIK), mixed numerical-analytical approaches [5] etc. Dealing with redundancy in IK although leads to infinite solutions for the joint space but offers greater flexibility and dexterity in motion as different constraint based or goal based criteria can be formulated as sub tasks in the solution. Two kinds of approaches have been reported in the literature to deal with this situation. One is set to exploit the null space of the Jacobian matrix in the homogeneous solution that infuses self motion of joints without affecting the task space. Typical method of this kind is gradient projection method (GPM) [6] and more recent [7]. In GPM the anti-gradient of a quadratic cost function, is projected in the null space of the task Jacobian, which is reminiscent of the projected gradient method for constrained minimization. The other is weighted least norm (WLN) approach [8], which minimizes the weighted norm of joint rate. In both the cases the primary task is to follow the prescribed trajectory and there may be multiple secondary tasks or nested subtasks with priority fixation [9][10].

The stability analysis of IK problem in discrete time domain comes in to picture when it requires implementation in hardware level as it provides useful guidelines for gain selection in relation to the sampling time. The approaches are mainly Lyapunov based [11]-[14] or non Lyapunov based using local exponential asymptotical stability condition [15].

This paper does not intend to propose any novel stability proof but investigates the stability conditions of first and second order redundant IK system which is formulated by augmenting the weighted least norm (WLN) solution. The WLN solution is augmented by weighted residual of the current joint rate and preferred pose rate in null space, so that we can arrive at a solution which is able to handle both joint limits and preferred joint configuration simultaneously satisfying the primary task, henceforth called as composite weighted least norm solution (CWLS). While doing this, the first and second order inverse kinematics solutions with redundancy have been re-casted as a feedback control problem, with the classical Close Loop Inverse Kinematics (CLIK) both for range space and null space and its stability conditions are derived for continuous and discrete time domains.

\section{INVERSE KINEMATICS PROBLEM}

The first and second order kinematics for the time variant task space defined as $x(t) \in \mathfrak{R}^{(m \times 1)}$ and joint space $q(t) \in \mathfrak{R}^{n \times 1}$ related by the direct kinematic non linear and transcendental vector function $k_{t}(q)$, whose time differentiation will define the non square analytic Jacobian matrix $J(q) \square J_{t}^{i j}(q) \square \partial k_{t}^{j} / \partial q_{i} \in \mathfrak{R}^{m \times n} ; \forall n>m$, with its assumption of bounded higher order terms and linearization. We denote the desired task space positions, velocities, and accelerations as $x_{d}, \dot{x}_{d}$ and $\ddot{x}_{d}$ respectively and reference joint configuration as $q_{r}$. Dropping the subscript $\mathrm{t}$ for brevity, the classical forward kinematics differential relationships can 
be expressed as

$$
\dot{x}=J(q) \dot{q} ; \text { and } \ddot{x}=J(q) \ddot{q}+\dot{J}(q, \dot{q}) \dot{q}
$$

and inverse kinematics least norm (LN) general solution as

$$
\begin{aligned}
& \dot{q}=\dot{q}_{p}+\dot{q}_{h}=J^{\dagger} \dot{x}+\left(I-J^{\dagger} J\right) \xi_{1} \\
& \ddot{q}=J^{\dagger}\left(\ddot{x}_{d}-\dot{J} \dot{q}\right)+\left(I-J^{\dagger} J\right) \xi_{2}
\end{aligned}
$$

where $\quad \dot{q}_{p} \in \mathfrak{R}(J)$ is particular solution, $\quad \dot{q}_{h} \in \mathfrak{N}(J)$ is homogeneous solution, $\quad J^{\dagger} \square J^{T}\left(J J^{T}\right)^{-1}$ is the right pseudoinverse of the Jacobian, $\xi_{1}$ and $\xi_{2} \in \mathfrak{R}^{n \times 1}$ are arbitrary vectors and $\left(I-J^{\dagger} J\right)$ is the null space projector. The Weighted Least Norm (WLN) solution formulates the problem as $\min (\dot{q})\left[\mathcal{H}_{1}(\dot{q})\right]=\min (\dot{q}) \square \dot{q} \square^{2}=\min (\dot{q})\left[\dot{q}^{T} W_{1} \dot{q}\right]$, st $(\dot{x}-J \dot{q})=0, \forall W_{1} \in \square^{n \times n}$ is the symmetric positive definite weighing matrix. To stabilize the ill posed condition of LN or WLN solution near singularities, Tikhonov like regularization has been used, which makes a trade off between tracking accuracy and the feasibility of the joint velocities, known as classical damped least square (DLS) solution. The trade off parameter is the damping factor is $\alpha$. If the objective is specified through a configuration rate dependent performance criteria $\mathcal{H}_{2}(\dot{q})$, set to be the closest to some particular pose, hence forth called the reference configuration $\left(q_{r}\right)$ the problem is reformulated as

$$
\begin{gathered}
\min (\dot{q})\left[\mathcal{H}_{2}(\dot{q})\right]=\min (\dot{q})\left[(1 / 2)\left(\dot{q}-\dot{q}_{r}\right)^{T} W_{2}\left(\dot{q}-\dot{q}_{r}\right)\right] \\
\text { s.t } J \dot{q}=\dot{x} ; \forall W_{2} \in \square^{n \times n}
\end{gathered}
$$

\subsection{Composite weighted least norm}

In our approach an augmented objective function has been formulated by combining configuration rate dependent performance criteria $\mathcal{H}_{2}(\dot{q})$ in Eq.(3) for pose optimization and $\mathcal{H}_{1}(\dot{q})$ for joint limit avoidance, subjected to the requirement of primary task space, as $\forall \mathcal{H}(\dot{q})=\mathcal{H}_{1}(\dot{q})+\mathcal{H}_{2}(\dot{q})$ and $\forall\left(W_{1}, W_{2}\right) \in \square^{n \times n}$, henceforth called as composite weighted least norm solution (CWLS) as,

$$
\begin{gathered}
\min (\dot{q}) \mathcal{H}(\dot{q})=\min (\dot{q})\left[(1 / 2) \dot{q}^{T} W_{1} \dot{q}\right. \\
\left.+(1 / 2)\left(\dot{q}-\dot{q}_{r}\right)^{T} W_{2}\left(\dot{q}-\dot{q}_{r}\right)\right] ; \text { s.t } J \dot{q}=\dot{x}
\end{gathered}
$$

To solve this optimization problem with equality constraint, it should satisfy both the necessary condition $\nabla_{\dot{q}} L=0$ and sufficient condition $\nabla_{\dot{q}}^{2} L>0$, where the Lagrangian is $L(\dot{q}, \lambda)=\mathcal{H}(\dot{q})+\lambda(J \dot{q}-\dot{x}) \quad$ and we can directly evaluate $\nabla_{\dot{q}}^{2} L=\left(W_{1}+W_{2}\right)>0$, which is true for minimization. Putting the value of $\dot{q}$ from $\nabla_{\dot{q}} L=0$ in the expression $\nabla_{\lambda} L=0$, we get $\lambda$. Substituting $\lambda$ back in $\dot{q}$ from $\nabla_{\dot{q}} L=0, \quad$ and $\quad \forall J^{\hbar} \square W^{-1} J^{T}\left(J W^{-1} J^{T}\right)^{-1}$, $\forall W \square\left(W_{1}+W_{2}\right), \forall \xi_{1} \square \dot{q}_{r}$, the general solution of CWLS reduces to [Appendix-I.A]

$$
\dot{q}=J^{\hbar} \dot{x}+\left(I-J^{\hbar} J\right) W^{-1} W_{2} \xi_{1}
$$

It is trivial to show $\left(I-J^{\hbar} J\right) W^{-1} W_{2}$ is the null space projector of reference joint rate vector $\dot{q}_{r}$ and hence no impact on task space as $J J^{\hbar}=I$. The optimization in the direction of the anti-gradient of a scalar configuration dependent performance criteria $\mathcal{H}_{3}(q)$ can also be set up by minimizing $\mathcal{H}_{3}(q)$ for weighted reference configuration $\left(q_{r}\right)$ as

$$
\begin{gathered}
\mathcal{H}_{3}(q)=(1 / 2)\left(q-q_{r}\right)^{T} W_{2}\left(q-q_{r}\right) \\
\Rightarrow \nabla_{q} \mathcal{H}_{3}(q)=W_{2}\left(q-q_{r}\right)
\end{gathered}
$$

$\forall \xi_{1}^{\prime} \square-k_{H}\left(W_{1}+W_{2}\right)^{-1} W_{2} \nabla_{q} \mathcal{H}_{3}(q)$, for a positive scalar $k_{H}$, the GPM flavor of CWLS formulation is

$$
\begin{gathered}
\dot{q}=J^{\hbar} \dot{x}+\left(I-J^{\hbar} J\right) W^{-1} W_{2} \xi_{1}^{\prime} \\
\ddot{q}=J^{\hbar}\left(\ddot{x}_{d}-\dot{J} \dot{q}\right)+\left(I-J^{\hbar} J\right) \xi_{2}
\end{gathered}
$$

Using, the relation $\dot{J} J^{\dagger}=-J \dot{J}^{\dagger}$ and after simplification we can establish the relation between $\xi_{2}$ and $\xi_{1}$ as.

$$
\xi_{2}=\dot{J}^{\dagger} J\left(\dot{q}-\xi_{1}\right)+\dot{\xi}_{1}
$$

\subsection{Control formulation}

Introducing proportional $\left(K_{P}\right)$ and derivative $\left(K_{D}\right)$ error control in Eq.(7) by positive definite diagonal gain matrices and task space error $e \square x_{d}-x=x_{d}-\kappa(q)$, we can arrive at the second order close loop kinematic scheme with task space error system

$$
\begin{gathered}
\ddot{q}=J^{h}\left(\ddot{x}_{d}-\dot{J} \dot{q}+K_{D} \dot{e}+K_{P} e\right)+\left(I-J^{h} J\right) \xi_{2} \\
\ddot{e}+K_{D} \dot{e}+K_{P} e=0
\end{gathered}
$$

In defining the null space controller, the first question that has to be answered is how many sub tasks the null space can simultaneously handle? If we choose $k$ sub tasks each of rank $r_{k}$, the limit is $\sum_{i=1}^{k} r_{i}=n$. Once all the dof's are exhausted, it is useless to put additional low priority tasks, as their contribution will be always projected in to null space or they can even corrupt the primary task. Dropping the regularizing term for the time being and defining the null space error as $e_{N}$, the null space contribution $\phi_{N}$ is

$$
\begin{gathered}
\phi_{N}=\left(I-J^{h} J\right)\left[\dot{\xi}_{1}+K_{N} e_{N}+K_{N D} \dot{e}_{N}-\dot{J}^{h} J\left(\xi_{1}-\dot{q}\right)\right] \\
\forall e_{N} \square\left(I-J^{h} J\right)\left(\xi_{1}-\dot{q}\right)
\end{gathered}
$$

with Proportional $\left(K_{N}\right)$ and Derivative $\left(K_{N D}\right)$ error control gain in null space.

\section{STABILITY}

A Lyapunov direct method argument can be used to analyze dependence $\dot{q}$ that ensures asymptotic stability of the error system in Eq.(9), as it associates an energy-based description with a (linear or nonlinear) autonomous system and its primary basis rests on the principle that for each system state with the exception of the equilibrium state, the time rate of such energy is negative, then energy decreases along any system trajectory until it attains its minimum at the equilibrium state; this argument justifies an intuitive concept of stability. 


\subsection{First order continuous time domain}

Choosen as Lyapunov function candidate $V(e)$ in positive definite quadratic form and $K_{p}, K_{N S}$ are the symmetric positive definite matrices,

$$
\begin{gathered}
V(e)=\frac{1}{2} e^{T} K_{p} e+V_{2} ; \text { where } V_{2}=\frac{1}{2} \beta^{2} \dot{q}^{T} K_{N S} \dot{q} \\
\dot{V}(e)=e^{T} K_{p} \dot{e}+\dot{V}_{2}=e^{T} K_{p}\left(\dot{x}_{d}-\dot{x}\right)+\dot{V}_{2}=e^{T} K_{p} \dot{x}_{d}-e^{T} K_{p} J \dot{q}+\dot{V}_{2} \\
=e^{T} K_{p} \dot{x}_{d}-e^{T} K_{p} J J^{h} \dot{x}_{d}-e^{T} K_{p} J J^{h} K_{p} e \\
\quad-e^{T} K_{p} J\left(I_{n \times n}-J^{\hbar} J\right) \xi_{1}+\dot{V}_{2} \\
=e^{T} K_{p}\left(I-J J^{h}\right) \dot{x}_{d}-e^{T} K_{p} J J^{h} K_{p} e \\
-e^{T} K_{p} J\left(I_{n \times n}-J^{\hbar} J\right) \xi_{1}+\dot{V}_{2} \\
\because J J^{h}=I ; \text { and } J\left(I_{n \times n}-J^{\hbar} J\right) \xi_{1}=\mathrm{O} \\
\dot{V}(e)=-e^{T} K_{p}^{T} K_{p} e+\dot{V}_{2}
\end{gathered}
$$

Considering the case of a constant reference $\left(\dot{x}_{d}=0\right)$, the function $\dot{V}(e)$ is negative definite, under the assumption of full rank for $\mathrm{J}$ and $\beta$ is so chosen such that $\dot{V}_{2}$ is negative.

\subsection{First order discrete time domain}

The approach in [12]-[15] which uses both Lyapunov and non Lyapunov methods, has been followed and implemented in CWLS solution for stability proof. Non Lyapunov methods prove the stability of the algorithm according to the comparison principle for discrete-time systems. The adopted methodology is not based on Lyapunov arguments, since to prove that the origin of the task space error space is asymptotically stable is not so trivial, because the Lyapunov function candidate depends not only on the task space but on the configuration variables too. Therefore, it cannot be shown to be positive definite without the inclusion of the terms that depend on the configuration variables [15].For sampling time $\mathrm{T}$, with proportional gain matrix $K_{p}$ in CLIK, forward kinematics relation $x_{k}=\kappa\left(q_{k}\right)$, the first order update rule for $(k+1)$ th time step is

$$
q_{k+1}=q_{k}+T J^{h} K_{p}\left(x_{d}-\kappa\left(q_{k}\right)\right)+T\left(I-J_{k}^{h} J_{k}\right) \xi_{1, k}
$$

With constant $x_{d}$, and as null space has no effect in task space error $(e)$, defining $r_{k}\left(\tilde{q}_{k}\right)=r_{k}\left(K_{p} T J_{k}^{h} e_{k}\right)$ as residual of Taylors series expansion of $\kappa\left(q_{k}+\tilde{q}_{k}\right)$, it can be proved that $\exists v_{k}>0 \square r_{k}(q) \square \leq v_{k} \square \tilde{q} \square^{2} ; \forall \tilde{q}: q+\tilde{q} \in \mathfrak{R}^{n \times 1}$. Neglecting higher order terms in linearization in the definition of Jacobian matrix, the dynamics of the task space error in Eq.(12) can be established as

$$
\begin{gathered}
e_{k+1}=e_{k}-K_{p} T J_{k} J_{k}^{h} e_{k}-r_{k}\left(\tilde{q}_{k}\right) \\
=\left(I-K_{p} T\right) e_{k}-r_{k}\left(\tilde{q}_{k}\right) \because J_{k} J_{k}^{h}=I
\end{gathered}
$$

In [15] it has been established that assuming $\square J^{\dagger} \square \leq \delta^{\prime}$, initial bounded task space error $\phi=e_{0}$ lies between $0<\left\|e_{0}\right\|<\frac{1}{K_{p} T v_{k} \delta^{\prime}}$ or $0<\left\|e_{0}\right\|<\frac{2-K_{p} T}{K_{p} T v_{k} \delta^{\prime}}$, and the gain between $0<K_{p} \leq 1 / T$. or $1 / T<K_{p} \leq 2 / T$, the least norm version of the CLIK algorithm ensures the exponential convergence of the task space error dynamics with

$$
\square e_{k} \llbracket \leq \phi \theta^{k} \quad \forall k \geq 0 ; \theta \in(0,1)
$$

We will use this relation in proving the discrete time convergence in CWLS solution. Using notation $\bar{\sigma}$ for $\max (\operatorname{svd}())$ and $\underline{\sigma}$ for $\min (\operatorname{svd}())$, as spectral norm is a function of $\bar{\sigma}$, form algebra of matrix norms we can define $\exists \bar{\sigma}_{w 1}>0 \unlhd W_{1} \llbracket=\bar{\sigma}_{w 1} ; \quad \exists \underline{\sigma}_{w 1}>0 \unlhd W_{1}^{-1} \sqsubset=\left(1 / \underline{\sigma}_{w 1}\right) ;$ $\exists \bar{\sigma}_{w 2}>0 \unlhd W_{2} \square=\bar{\sigma}_{w 2}, \quad \exists \underline{\sigma}_{w 2}>0 \unlhd W_{2}^{-1} \sqsubset=\left(1 / \underline{\sigma}_{w 2}\right)$, since $\left[W_{1}, W_{2}\right] \in \square^{\mathrm{n} \times \mathrm{n}}$ are symmetric positive definite and diagonal matrices resulting [ Appendix-I.B]

$$
\begin{gathered}
\left\|J^{h^{*}}\right\| \leq\left(\frac{\bar{\sigma}}{\underline{\sigma} \bar{\sigma}+\underline{\sigma}_{w} \alpha^{2}}\right) \square \delta_{1}^{\prime} \\
\text { and } \square J^{h} \square=\square J_{w}^{\dagger} \square=\square J^{\dagger} \square \frac{1}{\underline{\sigma}(J)} \square \delta_{2}^{\prime}
\end{gathered}
$$

where $\left\|J^{h^{*}}\right\| \square W^{-1} J^{T}\left(J W^{-1} J^{T}+\alpha^{2} I_{m \times m}\right)^{-1}$. Now we have to establish the bound of $q_{k+1}$. The bound of $q_{k+1}$ can be shown from Eq.(12) as

$$
\begin{gathered}
\square q_{k+1} \square \leq \square q_{k} \square+\square K_{p} T J_{k}^{h} e_{k} \square+\square T\left(I-J_{k}^{h} J_{k}\right) \xi_{1, k} \square \\
\quad \leq \square q_{k} \square+K_{p} T \delta_{2}^{\prime} \square e_{k} \square+T \square\left(I-J_{k}^{h} J_{k}\right) \square \xi_{1, k} \square
\end{gathered}
$$

$\because \square\left(I-J_{k}^{h} J_{k}\right) \sqsubset=1$ (Norm of null space projector is 1) and from Eq.(13)

$$
\begin{aligned}
& \square q_{k+1} \square \leq \square q_{k} \square+\left(K_{p} T \delta_{2}^{\prime}\right) \phi \theta^{k}+k_{H} K_{w} T \square q_{k}-q_{r, k} \square \\
& \leq \square q_{k} \square+\left(K_{p} T \delta_{2}^{\prime}\right) \phi \alpha^{k}+k_{H} K_{w} T \square q_{k} \square+k_{H} K_{w} T \square q_{r, k} \| \\
& \leq\left(1+k_{H} K_{w} T\right) \square q_{k} \square+k_{H} K_{w} T \square q_{r, k} \square+K_{p} T \delta_{2}^{\prime} \phi \theta^{k} \\
& \forall K_{n s} \square k_{H} K_{w} \text { (null space gain) } \\
& \square q_{k+1} \square \leq\left(1+K_{n s} T\right) \square q_{k} \square+K_{n s} T \square q_{r, k} \square+K_{p} T \delta_{2}^{\prime} \phi \theta^{k}
\end{aligned}
$$

The equivalent scalar liner system of Eq. (17) is

$$
\tilde{q}_{k+1}=\left(1+K_{n s} T\right) \tilde{q}_{k}+\left(K_{n s} T\right) \tilde{q}_{r, k}+K_{p} T \delta_{2}^{\prime} \phi \theta^{k}
$$

and its response $\forall k \geq 0$ with initial condition $\quad \tilde{q}_{0}=\square q_{i} \square$ and $\tilde{q}_{r 0}=\square q_{r i} \square$ is

$$
\tilde{q}_{k}=\left(1+K_{n s} T\right) \tilde{q}_{0}+\left(K_{n s} T\right) \tilde{q}_{r 0}+\frac{K_{p} T \delta_{2}^{\prime} \phi}{1-\theta}\left(1-\theta^{k}\right)
$$

$\because \theta<1,\left(1-\theta^{k}\right)$ will expontially converge to 1 , as $k \rightarrow \infty$, hence $\forall k \geq 0$

$$
\square q_{k} \square \leq \tilde{q}_{k} \leq\left(1+K_{n s} T\right) \tilde{q}_{0}+\left(K_{n s} T\right) \tilde{q}_{r 0}+\frac{K_{p} T \delta_{2}^{\prime} \phi}{1-\theta} \leq \rho
$$

From Eq.(16)

$$
\begin{aligned}
& \square q_{k+1}-q_{k} \square \leq \square q_{k+1} \square+\square q_{k} \square \\
& \leq\left(1+K_{n s} T\right) \square q_{k} \square+K_{n s} T \square q_{r, k} \square+K_{p} T \delta_{2}^{\prime} \phi \theta^{k}+\square q_{k} \square \\
& \leq\left(2+K_{n s} T\right) \square q_{k} \square+K_{n s} T \square q_{r, k} \square+K_{p} T \delta_{2}^{\prime} \square e_{k} \square \\
& \leq K_{p} T \delta_{2}^{\prime} \square e_{k} \square \leq K_{p} T \delta_{2}^{\prime} \phi \theta^{k} \\
& \quad \therefore \forall \theta<1 \text { and } k \geq 0 ; \square q_{k+1}-q_{k} \square \leq K_{p} T \delta_{2}^{\prime} \phi \theta^{k}
\end{aligned}
$$

$\because \forall \theta<1,\left(\theta^{k}\right)$ will exponentially converge to 0 , as $k \rightarrow \infty$, 


$$
\underset{\lim k \rightarrow \infty}{\square q_{k+1}-q_{k} \square=} \lim k \rightarrow \infty_{\operatorname{lom}}\left(K_{p} T \delta_{2}^{\prime} \phi\right) \theta^{k}=0
$$

This is true with the inclusion of an additional bounded condition for Null Space gain factor $K_{n s}$. Hence the bound for gains are,

$$
\begin{gathered}
0<K_{n s} \leq 1 / T \\
0<K_{p} \leq 1 / T \text { and } \square \mathrm{e}_{0} \square<\frac{1}{K_{p} T v_{k} \delta_{2}^{\prime}} \\
\text { or, } 1 / T<K_{p} \leq 2 / T \text { and } \square \mathrm{e}_{0} \square<\frac{2-K_{p} T}{K_{p} T v_{k} \delta_{2}^{\prime}}
\end{gathered}
$$

If $K_{p}$ is a positive definite (usually diagonal) matrix, the system is asymptotically stable. The error tends to zero along the trajectory with a convergence rate that depends on the eigenvalues of matrix $K_{p}$, the larger the eigenvalues, the faster the convergence. Since the scheme is practically implemented as a discrete-time system, it is reasonable to set an upper bound exists on the eigenvalues; depending on the sampling time $\mathrm{T},\left(0<K_{p} \leq 1 / T\right.$ and $\left.0<K_{n s} \leq 1 / T\right)$ along with initial error norm $\left(\square \mathrm{e}_{0} \square<1 / K_{p} T v_{k} \delta_{2}^{\prime}\right)$, there will be a limit for the maximum eigenvalue of $K_{p}$ under which asymptotic stability of the error system is guaranteed.

\subsection{Second order continuous time domain}

In second order analysis we will start with the regularized form of CWLS solution, considering $J^{\hbar}$ is the particular case of $J^{\hbar^{*}}, \forall \alpha=0, \forall J^{\hbar^{*}} \square W^{-1} J^{T}\left(J W^{-1} J^{T}+\alpha^{2} I\right)^{-1}$. We can set the error system with null space projector $\left(I-J^{\hbar} J\right)$ as

$$
\begin{aligned}
& \ddot{x}=J\left[J^{h^{*}}\left(\ddot{x}_{d}-\dot{J} \dot{q}+K_{D} \dot{e}+K_{P} e\right)+\left(I-J^{h} J\right) \xi_{2}\right]+\dot{J} \dot{q} \\
& =\left(I-J J^{h^{*}}\right)\left[\ddot{x}_{d}-\dot{J} \dot{q}+K_{D} \dot{e}+K_{P} e\right] ; \because J\left(I-J^{h} J\right)=O \\
& =N\left[\ddot{x}_{d}-\dot{J} \dot{q}+K_{D} \dot{e}+K_{P} e\right] ; \forall \mathbf{N} \square\left(I-J J^{h^{*}}\right)
\end{aligned}
$$

which reduces to the error differential system

$$
\ddot{e}+K_{D} \dot{e}+K_{P} e=\mathbf{N}\left[\ddot{x}_{d}-\dot{J} \dot{q}+K_{D} \dot{e}+K_{P} e\right]
$$

Introducing $\eta=\left[e^{T} \dot{e}^{T}\right]^{T}$ in Eq.(24), results in

$\dot{\eta}=\left[\begin{array}{c}\dot{e}^{T} \\ \ddot{e}^{T}\end{array}\right]=\left[\begin{array}{cc}0 & I \\ (N-I) K_{P} & (N-I) K_{D}\end{array}\right]\left[\begin{array}{c}e^{T} \\ \dot{e}^{T}\end{array}\right]+\left[\begin{array}{c}0 \\ N\left(\ddot{x}_{d}-\dot{J} \dot{q}\right)\end{array}\right]$ and $\forall \Phi \square(N-I)$

$\forall A \square\left[\begin{array}{cc}0 & I \\ \Phi K_{P} & \Phi K_{D}\end{array}\right] ; \quad$ and $\forall D \square\left[\begin{array}{c}0 \\ (I+\Phi)\left(\ddot{x}_{d}-\dot{J} \dot{q}\right)\end{array}\right]$, the error system can be reduces to

$$
\dot{\eta}=A \eta+D
$$

which describes a linear time invariant system with nonlinear state-dependent perturbation term and input disturbance. A Lyapunov direct method argument can be used to analyze stability of system in Eq.(25), as it associates an energy-based description with a (linear or nonlinear) autonomous system. Let the Lyapunov function candidate be

$$
\begin{gathered}
V(e)=\eta^{T} P \eta ; \forall P \square\left[\begin{array}{cc}
K_{P}+\rho K_{D} & \rho I \\
\rho I & I
\end{array}\right] \\
\text { and } \forall \rho \square \text { positive scalar }
\end{gathered}
$$

where positive definiteness of $V(e)$ is realized by the adoption of a quadratic form as $\mathrm{P}$ is a symmetric positive definite block-diagonal matrix and $V(e)>0, \forall e \neq 0$; $V(e)=0, \forall e=0$ and $V(e) \rightarrow \infty, \forall \mathrm{e} \rightarrow \infty$.Putting the values in the Lyapunov equation $A^{T} P+P A=-Q$, and after simplification reduces to

$$
-Q=\left[\begin{array}{cc}
2 \rho \Phi K_{P} & (I+\Phi)\left(K_{P}+\rho K_{D}\right) \\
(I+\Phi)\left(K_{P}+\rho K_{D}\right) & 2\left(\rho I+\Phi K_{D}\right)
\end{array}\right]
$$

Considering the case with out regularization $(\alpha=0)$, then $J^{\hbar^{*}}=J^{\hbar}, J J^{h}=I ; \quad N=O,(I+\Phi)=O$, Eq. (24) reduces to Eq. (9) and Eq.(27) reduces to

$$
Q=\left[\begin{array}{cc}
2 \rho K_{P} & O \\
O & 2\left(K_{D}-\rho I\right)
\end{array}\right] ; \because \Phi=-I
$$

which is symmetric positive definite block-diagonal matrix, as long as the arbitrary positive constant $\rho$ is smaller than the minimum element of the diagonal matrix $K_{D}$ i.e

$$
0<\rho<k_{D \min }
$$

For regularized composite weighted least norm ( RCWLN), $\Phi=(N-I)=\left(I-J J^{h^{*}}-I\right)=-J J^{h^{*}}$, and $I+\Phi=I-J J^{h^{*}}$. $\forall 0 \leq \alpha^{2} \leq \alpha^{2}{ }_{\max }$, the expression $\left(J W^{-1} J^{T}+\alpha^{2} I_{m \times m}\right)$ is always symmetric positive definite, since $J W^{-1} J^{T}$ is symmetric positive definite. This implies $J J^{h^{*}}=J(W)^{-1} J^{T}\left(J W^{-1} J^{T}+\alpha^{2} I_{m \times m}\right)^{-1}$ is symmetric positive definite, and $\Phi=-J J^{h^{*}}$ is always symmetric negative definite, resulting $\mathrm{Q}$ in Eq.(27) to be always symmetric positive definite. If $B \square J(W)^{-1} J^{T}$ and iff $\rho(B) \leq 1$, (for satisfying the convergence criteria) $J J^{h^{*}}=B\left(B+\alpha^{2} I_{m \times m}\right)^{-1} \approx B B^{-1}\left[I-\alpha^{2} B^{-1}\right] \approx\left[I-\alpha^{2} B^{-1}\right]$.

$\Rightarrow I+\Phi \approx \alpha^{2} B^{-1}$, which is symmetric positive definite. It does not affect the symmetric positive definiteness of $\mathrm{Q}$ in Eq.(27), being in minor diagonal of Q. Computing the time derivative of Eq.(26) we get

$$
\dot{V}(e)=-\eta^{T} Q \eta+2 \eta^{T} P k(e)
$$

Now the first term on the right-hand side of Eq.(30) is negative definite and the stability problem is reduced to searching a control law so that $k(e)$ renders the total $\dot{V}(e)$ negative (semi-)definite. Substituting $\eta, Q$ and $P$ in Eq.(30) and after expansion, rearranging we get

$$
\begin{gathered}
\dot{V}(e)=\left[2 \rho e^{T} \Phi K_{P} e+2 e^{T}(I+\Phi) K_{P} \dot{e}+2 \rho e^{T}(I+\Phi) K_{D} \dot{e}\right. \\
\left.+2 \dot{e}^{T}\left(\rho I+\Phi K_{D}\right) \dot{e}\right]+2\left(\rho e^{T}+\dot{e}^{T}\right)(I+\Phi)\left(\ddot{x}_{d}-\dot{J} \dot{q}\right)
\end{gathered}
$$


For CWLN solution without regularization, i.e $\forall J^{h^{*}}=J^{h}$ $\Phi=-I$ and $(I+\Phi)=O$,Eq.(31) reduces to

$$
\dot{V}(e)=-2\left[\rho e^{T} K_{P} e+\dot{e}^{T}\left(K_{D}-\rho I\right) \dot{e}\right]
$$

Eq. (32) is always negative definite $\forall J^{h}$ with the condition $0<\rho<k_{D \text { min }}$. Hence assuming at equilibrium, $e=0$, of the Composite Weighted Least Norm (CWLN) solution is asymptotically stable in Lyapunov sense.

For regularized CWLN solution there are contributions from $\|I+\Phi\|$, along with gain matrices, and also from maximum norm of desired end effector velocity and acceleration. We can derive the bound in terms of $W=\left(W_{1}+W_{2}\right)$ for RCWLS through singular value inequalities using the notation $\bar{\sigma} \square \max (\operatorname{svd}(J)), \underline{\sigma} \square \min (\operatorname{svd}(J)), \quad \bar{\sigma}_{w} \square \max (\operatorname{svd}(W))$ and $\underline{\sigma}_{w} \square \min (\operatorname{svd}(W))$, resulting [Appendix-I.C]

$$
\forall \alpha^{2}>0 ; \quad \square I+\Phi \square \leq\left(\frac{\bar{\sigma}_{w} \alpha^{2}}{\underline{\sigma}^{2}+\bar{\sigma}_{w} \alpha^{2}}\right) \square \beta^{\prime}
$$

and $\beta^{\prime}>0 \because[\bar{\delta}, \underline{\delta}]>0 ;\left[\underline{\sigma}_{w 1}, \underline{\sigma}_{w 2},\right]>0 ;\left[\bar{\sigma}_{w 1} \bar{\sigma}_{w 2,}\right]>0 ; \alpha>0$.

For estimation of bounds of the term $\|\dot{J} \dot{q}\|$, writing the ith component of the vector $v \square \dot{J} \dot{q}$, in the quadratic form as

$$
\begin{gathered}
v_{i}=\dot{q}^{T} \frac{\partial J_{i}(q)}{\partial q} \dot{q}=\dot{q}^{T} N_{i}(q) \dot{q}, \\
\forall N_{i}(q) \square \frac{\partial J_{i}(q)}{\partial q} \text { and } \dot{J}(q, \dot{q})=\frac{\partial J_{i}(q)}{\partial q} \frac{\partial q}{\partial t}
\end{gathered}
$$

we can express

$$
\begin{gathered}
\|v\|=\left\|\dot{q}^{T} N_{i}(q) \dot{q}\right\| \leq\left\|\dot{q}^{T}\right\|\left\|N_{i}(q)\right\|\|\dot{q}\| \\
\leq\left\|\dot{x}^{T} J^{h^{*} T}\right\|\left\|N_{i}(q)\right\|\left\|J^{h^{* T}} \dot{x}\right\|
\end{gathered}
$$

and deriving the expressions for the terms $\left\|J^{h}\right\|$, $\gamma_{h} \square\left\|J^{h^{*}}\right\|$ [ Appendix-I.B] and $\gamma \square\left\|N_{i}(q)\right\|$, we can finally establish

$$
\|v\| \square\left\|\dot{J}^{h^{*}} \dot{q}\right\| \leq\left(\frac{\bar{\sigma}}{\underline{\sigma} \bar{\sigma}+\underline{\sigma}_{w} \alpha^{2}}\right)^{2} \gamma\|\dot{x}\|^{2} \leq \gamma_{h}^{2} \gamma\|\dot{x}\|^{2}
$$

If $W 1=W 2=I$ in Eq.(34), $\gamma_{h}$ reduces to Regularized Least Norm and along with this, if $\alpha^{2}=0$, it reduces to Least Norm with $\left\|J^{h}\right\|=\left\|J^{\dagger}\right\|=\gamma^{\dagger}=1 / \underline{\sigma}$.Putting the relation $\dot{x}=\left(\dot{x}_{d}-\dot{e}\right)$ in Eq.(34) and $\forall \bar{v}_{d} \square$ as maximum norm of end effector velocity, we can express

$$
\|v\| \leq \gamma_{h}^{2} \gamma\left(\bar{v}_{d}^{2}+2 \bar{v}_{d}\|\dot{e}\|+\|\dot{e}\|^{2}\right)
$$

Rewriting the upper bound of Eq.(31) in terms of $\beta^{\prime}$ in Eq.(33), $\forall \bar{a}_{d} \square\left\|\ddot{x}_{d}\right\|, \kappa_{p} \square\left\|K_{P}\right\|, \kappa_{d} \square\left\|K_{D}\right\|$

$\|\dot{V}\| \leq-\rho\left(\kappa_{p}-\beta^{\prime} \kappa_{p}\right)\|e\|^{2}-\left[\left(\kappa_{d}-\beta^{\prime} \kappa_{d}\right)-\rho\right]\|\dot{e}\|^{2}$

$+\beta^{\prime}\left(\kappa_{p}+\rho \kappa_{d}\right)\|e\|\|\dot{e}\|+2 \rho \beta^{\prime} \bar{v}_{d} \gamma_{h}^{2} \gamma\|e\|\|\dot{e}\|+2 \beta^{\prime} \bar{v}_{d} \gamma_{h}^{2} \gamma\|\dot{e}\|^{2}$

$+\rho \beta^{\prime}\left(\bar{a}_{d}+\bar{v}_{d}^{2} \gamma_{h}^{2} \gamma\right)\|e\|+\left(\rho \beta^{\prime} \gamma_{h}^{2} \gamma\|e\|+\beta^{\prime} \gamma_{h}^{2} \gamma\|\dot{e}\|\right)\|\dot{e}\|^{2}$

$+\beta^{\prime}\left(\bar{a}_{d}+\bar{v}_{d}^{2} \gamma_{h}^{2} \gamma\right)\|\dot{e}\|$
Substituting $\quad \alpha_{p} \square \rho\left(\kappa_{p}-\beta^{\prime} \kappa_{p}\right), \quad \alpha_{p d} \square 2 \beta^{\prime}\left(\kappa_{p}+\rho \kappa_{d}\right)$, $\alpha_{d} \square\left(\kappa_{d}-\beta^{\prime} \kappa_{d}\right)-\rho, \kappa_{0} \square \beta^{\prime}\left(\bar{a}_{d}+\bar{v}_{d}^{2} \gamma_{h}^{2} \gamma\right), \kappa_{1} \square 2 \beta^{\prime} \bar{v}_{d} \gamma_{h}^{2} \gamma$ and $\kappa_{2} \square \beta^{\prime} \gamma_{h}^{2} \gamma$, after rearrangement Eq.(36) can be expressed as

$$
\begin{array}{r}
\|\dot{V}\| \leq-\alpha_{p}\|e\|^{2}+\alpha_{p d}\|e\|\|\dot{e}\|-\alpha_{d}\|\dot{e}\|^{2} \\
+(\rho\|e\|+\|\dot{e}\|)\left(\kappa_{0}+\kappa_{1}\|\dot{e}\|+\kappa_{2}\|\dot{e}\|^{2}\right)
\end{array}
$$

which can be rearranged to

$$
\begin{gathered}
\|\dot{V}\| \leq z^{T} G z+\left[\begin{array}{ll}
\rho & 1
\end{array}\right] z^{T}\left[\kappa_{0}+\left[\begin{array}{ll}
0 & \kappa_{1}
\end{array}\right] z^{T}+\left[\begin{array}{ll}
0 & \kappa_{2}
\end{array}\right] z^{2 T}\right. \\
\forall z \square\left[\frac{\|e\|}{\|\dot{e}\|}\right] ; G \square\left[\begin{array}{cc}
-\alpha_{p} & \alpha_{p d} / 2 \\
\alpha_{p d} / 2 & -\alpha_{d}
\end{array}\right]
\end{gathered}
$$

The $z^{T} G z$ term enforces the condition for positive determinant $(\operatorname{det}(G))$

$$
\forall \alpha_{p}>0 ; \forall \alpha_{d}>0 ; \alpha_{p} \alpha_{d}>\alpha_{p d}^{2} / 4
$$

which puts a upper positive bound for $\beta^{\prime}$

$$
\beta^{\prime}<1 \text { and } \beta^{\prime}<1-\rho / \kappa_{d}
$$

Defining [Appendix-I.D]

$$
\lambda_{1}\|\| G \| / \sqrt{1+\rho^{2}}=\frac{\left(\alpha_{p}+\alpha_{d}\right)+\sqrt{\left(\alpha_{p}^{2}+\alpha_{d}^{2}-2 \alpha_{p} \alpha_{d}+\alpha_{p d}^{2}\right)}}{2 \sqrt{1+\rho^{2}}}
$$

Eq.(38) can be rewritten as

$$
\begin{aligned}
& \|\dot{V}\| \leq\left\|\left[\begin{array}{ll}
\rho & 1
\end{array}\right]\right\|\left\|z^{T}\right\| \\
& {\left[\begin{array}{ll}
\kappa_{0}+ & \left\|\left[\begin{array}{ll}
0 & \kappa_{1}-\lambda_{1}
\end{array}\right]\right\|\|z\|+\left\|\left[\begin{array}{ll}
0 & \kappa_{2}
\end{array}\right]\right\|\|z\|^{2}
\end{array}\right]} \\
& \quad \leq\left(\sqrt{1+\rho^{2}}\right)\left\|z^{T}\right\|\left(\kappa_{0}+\left(\kappa_{1}-\lambda_{1}\right)\|z\|+\kappa_{2}\|z\|^{2}\right)
\end{aligned}
$$

$$
\begin{array}{r}
\Rightarrow \forall \Gamma\left(\left\|z^{T}\right\| \square\left(\kappa_{0}+\left(\kappa_{1}-\lambda_{1}\right)\|z \quad\|+\kappa_{2}\|z\|^{2}\right)\right. \\
\|\dot{V}\| \leq\left(\sqrt{1+\rho^{2}}\right)\left\|z^{T}\right\| \Gamma\left(\left\|z^{T}\right\|\right)
\end{array}
$$

Considering $\Gamma\left(\left\|z^{T}\right\|\right)=0$,

$$
\zeta_{1,2}=\frac{-\left(\kappa_{1}-\lambda_{1}\right) \pm \sqrt{\left(\kappa_{1}-\lambda_{1}\right)^{2}-4 \kappa_{0} \kappa_{2}}}{2 \kappa_{2}}
$$

$\Rightarrow \forall$ real and distinct roots $\left(\zeta_{1}, \zeta_{2}\right)$ of $\Gamma\left(\left\|z^{T}\right\|\right)=0$

$$
\left(\kappa_{1}+\lambda_{1}\right)^{2} \geq 4 \kappa_{0} \kappa_{2} \Rightarrow \lambda_{1} \geq \kappa_{1}+2 \sqrt{\kappa_{0} \kappa_{2}}
$$

$\Rightarrow \dot{V}(e)$ is upper bounded by a negative definite function in the region

$$
\Omega=\left\{\|z\|: \zeta_{1} \leq\|z\| \leq \zeta_{2}\right\}
$$

$\Rightarrow$ If the initial error norm is bounded, i.e $\left\|z_{0}\right\| \leq \zeta_{2} \forall T=T_{0}, \quad \exists \varepsilon>0 \quad$ and $\quad \exists T_{\varepsilon}>0$ so that $\left\|z_{t}\right\|-\zeta_{1} \leq \varepsilon \quad \forall T=T_{\varepsilon}+T_{0}$. In the case the initial error norm is $\left\|z_{0}\right\| \leq \zeta_{1}$, then $\dot{V}(e) \leq 0$ may not be negative and the error norm will rise. Hence we can conclude [i] $\forall\left\|z_{0}\right\| \leq \zeta_{2}$,

$\dot{V}(e) \leq 0 \quad \forall e \neq 0 \quad$ i.e negative definite . [ii] The norm of the error $(\|z\|)$ is bounded both for $\left\|z_{0}\right\| \leq \zeta_{2}$ and $\left\|z_{0}\right\| \leq \zeta_{1}$. Hence Regularized Composite Weighted Least Norm (RCWLN) solution is asymptotically stable in Lyapunov sense. 

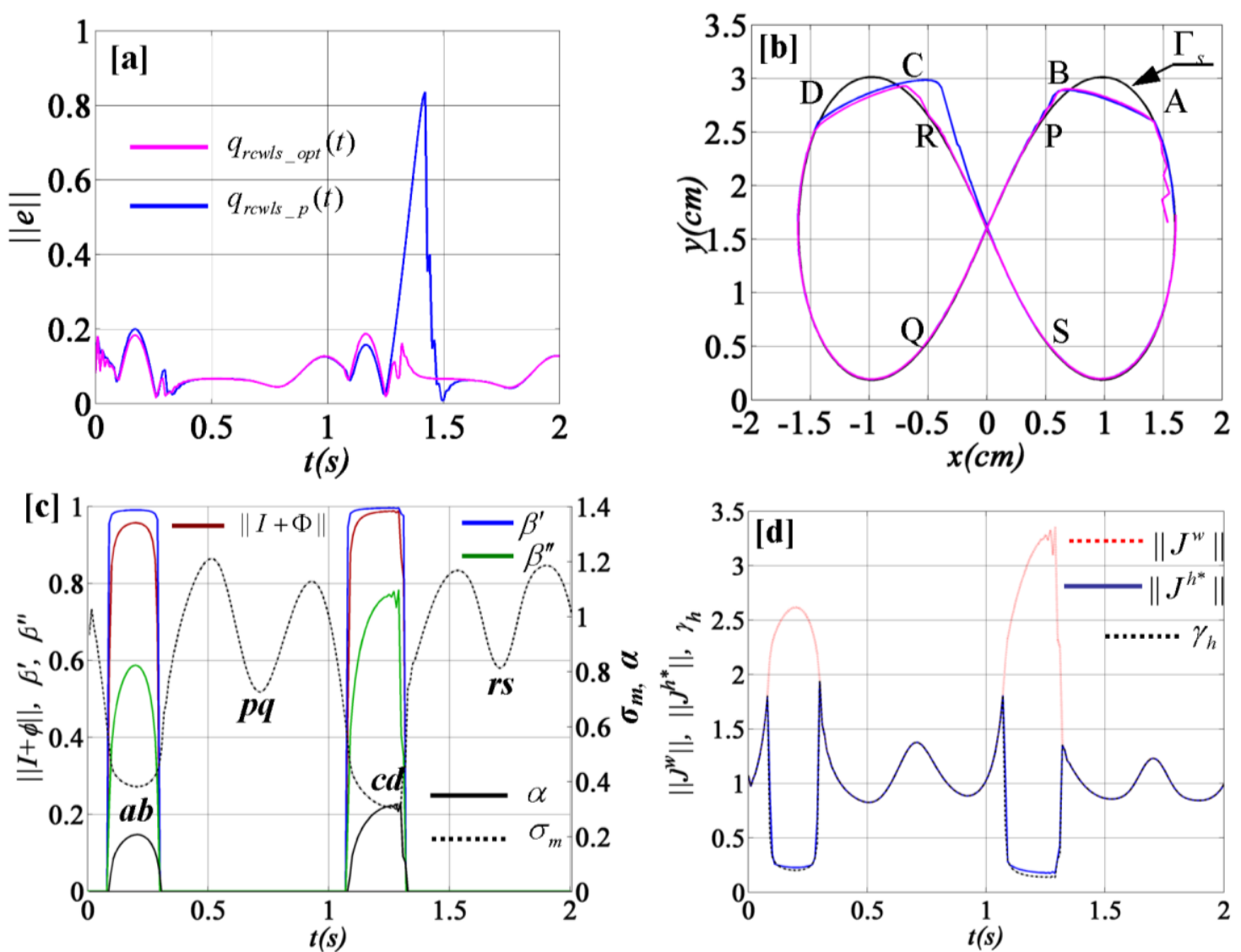

Figure-1: [a]: Time history of $\|e\|$ for lamniscate trajectory $\Gamma_{s}$. .b] Trajectory trace for the solutions. Analytical trajectory generating workspace singularity $\Gamma_{s}$ is ABPQDCRSA. [c] Time histories for $\|I+\Phi\|, \beta^{\prime}, \beta^{\prime \prime}$ on left $\mathrm{Y}$ axis and $\alpha, \sigma_{m} \square \min \operatorname{svd}(J)$ on right Y-axis. [d] Time histories for $J^{w} \square J_{w}^{\dagger}, J^{h^{*}}, \gamma_{h}$.

\section{DISCUSSIONS}

The above stability analysis results in getting relations among various system parameters. For a given trajectory if the upper bounds of the end effector velocity $\left(\bar{v}_{d}\right)$ and acceleration $\left(\bar{a}_{d}\right)$ and estimates of $[\underline{\sigma}, \bar{\sigma}]$ are known, then the estimates of $\gamma, \gamma_{h}$ and $\beta^{\prime}$ can be sought. Then it is possible to choose different parameters like damping factor $(\alpha)$, feedback gains $\left(K_{P}, K_{D}\right)$ etc, weighing matrix $\left(\mathrm{W}_{1}, \mathrm{~W}_{2}\right)$, and positive scalar $(\rho)$ based on the relations in Eq.(29), Eq(39), Eq.(40) and Eq.(43).

To illustrate the performance, we discuss the results of null

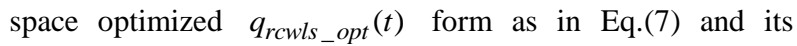
particular solution $q_{r c w l s_{-}}(t)$, for a planar serial 3RRR manipulator following a lamniscate trajectory $\left(\Gamma_{s}\right)$, crossing both workspace and configuration singularities. In both cases the regularized version is used. The link parameters in Denavit Hardenberg standard convention are $l_{i}=[1.5,0.9,0.7] \mathrm{cm}, \alpha_{i}=[0,0,0], d_{i}=[0,0,0] \quad$ and $\theta_{i}=\left[q_{1}, q_{2}, q_{3}\right] . \quad$ The iteration started with $W_{1}=I_{3 \times 3}$, $W_{2}=\operatorname{diag}[75.075 .075 .0], \quad K_{P}=\operatorname{diag}[4545]$, $K_{D}=\operatorname{diag}[0.450 .45]$, and $K_{I}=\operatorname{diag}[0.10 .1]$. The null space controller parameters are $k_{H}=0.95$, $K_{N P}=\operatorname{diag}[454545], \quad K_{N D}=\operatorname{diag}\left[\begin{array}{lll}2.5 & 2.5 & 2.5\end{array}\right], \quad$ and
$K_{N I}=\operatorname{diag}[1.01 .01 .0]$. The simulation time is $2 \mathrm{~s}$ with increment $d t=0.01 \mathrm{~s}$.

The first workspace singularity crossing occurs between $0.08 s \leq t \leq 0.3 s$ when the tip crosses from $\mathrm{A}$ to $\mathrm{B}$ in $\Gamma_{s}$ (Figure-1[b]) and second workspace singularity occurs between $1.1 s \leq t \leq 1.5 s$ when the tip crosses from $\mathrm{C}$ to $\mathrm{D}$. In between these two, the solution faces near configuration singularity when it crosses from $\mathrm{P}$ to $\mathrm{Q}$ between $0.6 s \leq t \leq 0.8 s$ and from $\mathrm{R}$ to $\mathrm{S}$ between $1.6 s \leq t \leq 1.8 s$. It is to be mentioned here that initial high oscillating acceleration between $0.0 s \leq t \leq 0.05 s$ in $\|e\|$, in Figure-1[a], is due to the task space gains. $\|e\|$ in $q_{\text {rcwls }}$ opt $(t)$ is considerably lower than that of $q_{r c w l s} p(t)$ when crossing the workspace singularity $D \rightarrow C$ (Figure-1[a]). In the near configuration singularity cases (pq and rs) in Figure-1[c] which lowers $\sigma_{m}(t)$, the damping parameter $\alpha(t)$ does not interfere $\forall \varepsilon=0.5$, the threshold value to initiate damping and $\alpha(t)=f\left(\sigma_{m}, \varepsilon\right)$.

Although $\left\|J^{h^{*}}\right\|$ is bounded by $\gamma_{h}$, Figure-1[d], , the RCWLN solution is also very sensitive to the gain of the positive definite weighing matrix $W$ or $\underline{\sigma}_{w}$ value since high gain $W_{1}$ steeply lowers $\bar{\sigma}_{w}$ and $\underline{\sigma}_{w}$ in $\gamma_{h}$ ( Figure$1[\mathrm{~d}])$ and hence the damping factor $\alpha$ is to be related to the 
estimate $\underline{\sigma}_{w}$ of the Jacobian for a particular time step to confine its role in reducing the potentially high norm joint rates near singularity positions. This implies that there must defined policies to separate the role of $W_{1}, W_{2}$ and $\alpha$ in RCWLN solution. For example in the case when the solution is away both from joint limits and singularity, the value of damping parameter alpha will be zero and the predominant role will be played by null space gain in the homogeneous solution as in Eq.(7). Since starting with $\left[W_{2}, W_{1}\right] \in I$, $\forall\left\|W_{2}\right\| \square\left\|W_{1}\right\|, \quad\left\|\left(W_{1}+W_{2}\right)^{-1} W_{2}\right\| \rightarrow 1$ and the contribution form null space will be maximum. This can be advantageously used as $\left(\dot{q}_{r}\right)$ will provide the information of feasible and unfeasible directions along which joint space solution will move in self motion as it does not has any effect in the task space. Again when the solution is approaching the joint limit, $\left\|W_{1}\right\| \rightarrow \infty$, keeping $\left\|W_{1}\right\|$ constant, $\left\|\left(W_{1}+W_{2}\right)^{-1}\right\| \rightarrow 0$, which will drastically reduce the null space contribution. The task space will remain unaffected as. , when $\alpha=0,\left\|J^{h}\right\|$ is independent of $W$.

From Eq.(40), $\max \left(\beta^{\prime}\right) \approx 1$ for scalar $\rho$ (Figure-1[c]). Also $\beta^{\prime}$ closely follows $\|I+\Phi\|$, than the formulation $\beta^{\prime \prime} \square \alpha^{2}\left(\underline{\sigma}^{2}+\alpha^{2}\right)$ as in Figure-1[c]. When approaching a singularity, the minimum singular value of the Jacobian $(\underline{\sigma})$ decreases and in order to keep $\beta^{\prime}$ near about unity, value of $\sigma_{w} \alpha^{2} \square 1$ should be high. As $\|W\|$ is already high in the formulation due to high gain of $\left\|W_{2}\right\|$, resulting high $\underline{\sigma}_{w}$, it is in coherence with the formulation as, very high value of $\alpha^{2}$ is not required and we can preset the $\alpha_{\max }^{2}=0.5$. This will avoid in generating high joint rates, of course at the cost of task space error.

\section{CONCLUSION}

Stability conditions in redundancy resolution of a solution augmenting weighted least norm solution by weighted residual of the current joint rate and preferred pose rate in null space have been derived for continuous and discrete time domains using Lyapunov and non Lyapunov based stability criteria. For generalization regularized version has been dealt with considering the null space contribution which will provide the information of feasible and unfeasible directions which is especially important in near singularity configuration. The relations among the parameters $\beta^{\prime}, \beta^{\prime \prime},\|I+\Phi\|, \underline{\sigma}_{w}, \alpha, \gamma_{h}$ etc obtained during the stability analysis can be verified from Figure-1[a]-[d], and valid only when the solution is approaching configuration or workspace singularity. During workspace singularity crossings $A \rightarrow B$ and $C \rightarrow D$, they does not hold good any more and the stability of $\|e\|$ drifted but the task space and null space controllers recovers the solution and brings the task space error back to its stability zone.

\section{ACKNOWLEDGMENTS}

The authors would like to acknowledge Council of Scientific and Industrial Research (CSIR), New Delhi, grant ESC-0203/04 for carrying out the work.

\section{REFERENCES}

[1] D. Whitney. 1969. Resolved motion rate control of manipulators and human prostheses. IEEE Trans. ManMach. Syst., vol. MMS-10, no. 2, pp. 47-52.

[2] A. Goldenberg, B. Benhabib, and R. Fenton. 1985.A complete generalized solution to the inverse kinematics of robots. IEEE J. Robot. Autom., vol. RA-1, no. 1, pp. 14-20.

[3] J. Zhao and N. Badler. 1994. Inverse kinematics positioning using nonlinear programming for highly articulated figures. Trans. Computer Graph. vol. 13, no. 4, pp. 313-336.

[4] L. Sciavicco and B. Siciliano. 1986. Coordinate transformation: A solution algorithm for one class of robots. IEEE Trans. Syst., Man, Cybern., vol. SMC-16, no. 4 , pp. 550-559.

[5] M. Tarokh and M. Kim. 2007. Inverse kinematics of 7DOF robots and limbs by decomposition and approximation. IEEE Trans. Robot., vol. 23, no. 3, pp. 595-600.

[6] A. Liëgeois. 1977. Automatic supervisory control of the configuration and behavior of multibody mechanisms. IEEE Transactions on Systems, Man, and Cybernetics, SMC-7(12):868-871.

[7] N. Mansard and F. Chaumette. 2009. Directional redundancy for robot control. IEEE Transactions on Automatic Control, 54(6):1179-1192.

[8] T. F. Chan and R. V. Dubey. 1995. A weighted leastnorm solution based scheme for avoiding joint limits for redundant joint manipulators. IEEE Transactions on Robotics and Automation, 11(2):286-292.

[9] Y. Nakamura, H. Hanafusa, T. Yoshikawa. 1987. TaskPriority Based Control of Robot Manipulators. International Journal of Robotics Research, Vol. 6, No. 2 , pp. $3-15$.

[10] Oussama Kanoun, Florent Lamiraux, Pierre-Brice Wieber. 2011. Kinematic Control of Redundant Manipulators: Generalizing the Task-Priority Framework to Inequality Task. IEEE Transactions on Robotics 27(4): 785-792.

[11] H.Das, J.-J. Slotine, and T. Sheridan. Inverse kinematic algorithms for redundant systems. in Proc. IEEE Int. Conf. Robot. Autom., San Francisco, CA, 1988, pp. 4348 .

[12] G. De Maria and R. Marino. A discrete algorithm for solving the inverse kinematic problem for robotic manipulators. Proc. 2nd Int. Conf. Adv.Robot., Tokyo, Japan, 1985, pp. 275-282.

[13] G. Antonelli. 2009. Stability analysis for prioritized closed-loop inverse kinematic algorithms for redundant robotic systems. IEEE Trans. Robot., vol. 25, no. 5, pp. 985-994.

[14] Fabrizio Caccavale, Stefano Chiaverini, and Bruno Siciliano. 1997. Second-Order Kinematic Control of Robot Manipulators with Jacobian Damped LeastSquares Inverse: Theory and Experiments.", IEEE/ASME Transactions on Mechatronics, vol. 2, no. 3. 
[15] P. Falco, C. Natale. 2011. On the stability of closed-loop inverse kinematics algorithms for redundant robots", IEEE Trans. on Robotics (short paper), IEEE Press, Inc., vol. 27, No. 4, pp. 780-784.

\section{APPENDIX- I.A}

Lagrangian $: L(\dot{q}, \lambda)=\mathcal{H}(\dot{q})+\lambda(J \dot{q}-\dot{x})$

$=\left[(1 / 2) \dot{q}^{T} W_{1} \dot{q}+(1 / 2)\left(\dot{q}-\dot{q}_{r}\right)^{T} W_{2}\left(\dot{q}-\dot{q}_{r}\right)\right]+\lambda(J \dot{q}-\dot{x})$

$\nabla_{\dot{q}} L=\frac{\partial L^{T}}{\partial \dot{q}} \Rightarrow W_{1} \dot{q}+W_{2}\left(\dot{q}-\dot{q}_{r}\right)+J^{T} \lambda=0$

and $\nabla_{\lambda} L=J \dot{q}-\dot{x}=0$ with $\nabla_{\dot{q}}^{2} L=\left(W_{1}+W_{2}\right)>0$

$\Rightarrow \dot{q}=\left(W_{1}+W_{2}\right)^{-1}\left(W_{2} \dot{q}_{r}-J^{T} \lambda\right)$

Putting the value of $\dot{q}$ in $\nabla_{\lambda} L=0$

$\lambda=\left(J W^{-1} J^{T}\right)^{-1}\left[J W^{-1} W_{2} \dot{q}_{r}-\dot{x}\right] ; \forall W \square\left(W_{1}+W_{2}\right)$

$\Rightarrow \dot{q}=\left(W_{1}+W_{2}\right)^{-1}\left(W_{2} \dot{q}_{r}-J^{T} \lambda\right)$

Putting the value of $\dot{q}$ in $\nabla_{\lambda} L=0$

$\lambda=\left(J W^{-1} J^{T}\right)^{-1}\left[J W^{-1} W_{2} \dot{q}_{r}-\dot{x}\right] ; \forall W \square\left(W_{1}+W_{2}\right)$

$\Rightarrow \dot{q}=W^{-1} J^{T}\left(J W^{-1} J^{T}\right)^{-1} \dot{x}+\left(I-W^{-1} J^{T}\left(J W^{-1} J^{T}\right)^{-1} J\right) W^{-1} W_{2} \dot{q}_{r}\left\|\alpha^{2} B A^{-1}\right\| \leq\left|\alpha^{2}\right|\|\mathrm{B}\|\left\|A^{-1}\right\| ; \because A^{-1}=W \Rightarrow\left\|A^{-1}\right\|=\bar{\sigma}(W)=\bar{\sigma}_{w}$ $\forall J^{\hbar} \square W^{-1} J^{T}\left(J W^{-1} J^{T}\right)^{-1} ; \forall \xi_{1} \square\left(W_{1}+W_{2}\right)^{-1} W_{2} \dot{q}_{r}$ $\dot{q}=J^{\hbar} \dot{x}+\left(I-J^{\hbar} J\right)\left(W_{1}+W_{2}\right)^{-1} W_{2} \dot{q}_{r}=J^{\hbar} \dot{x}+\left(I-J^{\hbar} J\right) \xi_{1}$

\section{APPENDIX- I.B}

$\left\|J^{h^{*}}\right\|=\bar{\sigma}\left[W^{-1} J^{T}\left(J W^{-1} J^{T}+\alpha^{2} I_{m \times m}\right)^{-1}\right]$;

$\leq \bar{\sigma}\left(W^{-1} J^{T}\right) \bar{\sigma}\left(J W^{-1} J^{T}+\alpha^{2} I_{m \times m}\right)^{-1} \because \bar{\sigma}(A B) \leq \bar{\sigma}(A) \bar{\sigma}(B) ;$

$\left\|J^{h^{*}}\right\| \leq \bar{\sigma}\left(W^{-1}\right) \bar{\sigma}(J) \frac{1}{\sigma\left(J W^{-1} J^{T}+\alpha^{2} I_{m \times m}\right)}$

$\because \bar{\sigma}\left(W^{-1}\right)=1 / \underline{\sigma}(W)$ and $\underline{\sigma}(A+B) \leq \underline{\sigma}(A)+\bar{\sigma}(B)$

$\underline{\sigma}(A B) \leq \underline{\sigma}(A) \bar{\sigma}(B)$

$\left\|J^{h^{*}}\right\| \leq \bar{\sigma}\left(W^{-1}\right) \bar{\sigma}(J) \frac{1}{\underline{\sigma}\left(J W^{-1} J^{T}\right)+\bar{\sigma}\left(\alpha^{2} I_{m \times m}\right)}$

$\left\|J^{h^{*}}\right\| \leq \frac{\bar{\sigma}(J)}{\underline{\sigma}(W)}\left(\frac{1}{\underline{\sigma}\left(J W^{-1}\right) \bar{\sigma}\left(J^{T}\right)+\bar{\sigma}\left(\alpha^{2} I_{m \times m}\right)}\right)$

$\left\|J^{h^{*}}\right\| \leq \frac{\bar{\sigma}(J)}{\underline{\sigma}(W)}\left(\frac{1}{\left.\underline{\sigma}(J) \bar{\sigma}\left(W^{-1}\right) \bar{\sigma}\left(J^{T}\right)+\alpha^{2}\right)}\right) ;$

$\left\|J^{h^{*}}\right\| \leq \frac{\bar{\sigma}(J)}{\underline{\sigma}(W)}\left(\frac{\underline{\sigma}(W)}{\left.\underline{\sigma}(J) \bar{\sigma}\left(W^{-1}\right) \bar{\sigma}\left(J^{T}\right)+\underline{\sigma}(W) \alpha^{2}\right)}\right)$

$\left\|J^{h^{*}}\right\| \leq\left(\frac{\bar{\sigma}}{\underline{\sigma} \bar{\sigma}+\underline{\sigma}_{w} \alpha^{2}}\right)$

\section{APPENDIX- I.C}

$$
\begin{aligned}
& \square I+\Phi \square=\square I-J J^{h^{*}} \square=\square I-J W^{-1} J^{T}\left(J W^{-1} J^{T}+\alpha^{2} I\right)^{-1} \| \\
& =\square I-\left(U \Sigma V^{T}\right)\left(U^{\prime} \Sigma_{w} U^{\prime T}\right)^{-1}\left(U \Sigma V^{T}\right)^{T} \\
& \ldots\left[\left(U \Sigma V^{T}\right)\left(U^{\prime} \Sigma_{w} U^{\prime T}\right)^{-1}\left(U \Sigma V^{T}\right)^{T}+\alpha^{2} I\right]^{-1} \| \\
& =\square I-\left(U \Sigma V^{T}\right)\left(U^{\prime} \Sigma_{w}{ }^{-1} U^{\prime T}\right)\left(V \Sigma U^{T}\right) \\
& \ldots\left[\left(U \Sigma V^{T}\right)\left(U^{\prime} \Sigma_{w}{ }^{-1} U^{\prime T}\right)\left(V \Sigma U^{T}\right)+\alpha^{2} U U^{T}\right]^{-1} \| \\
& =\square I-\left(U \Sigma V^{T}\right)\left(U^{\prime} \Sigma_{w}{ }^{-1} U^{\prime T}\right)\left(V \Sigma U^{T}\right) \\
& \ldots\left[U \Sigma V^{T}\left(U^{\prime} \Sigma_{w}{ }^{-1} U^{\prime T}+\alpha^{2} V \Sigma^{-2} V^{T}\right) V \Sigma U^{T}\right]^{-1} \|
\end{aligned}
$$

$=\square I-\left(U \Sigma V^{T}\right)\left(U^{\prime} \Sigma_{w}{ }^{-1} U^{\prime T} V \Sigma U^{T} U \Sigma^{-1} V^{T}\left(U^{\prime} \Sigma_{w}{ }^{-1} U^{\prime T}\right.\right.$

$\left.+\alpha^{2} V \Sigma^{-2} V^{T}\right)^{-1} V \Sigma^{-1} U^{T} \|$

$=\square I-\left(U \Sigma V^{T}\right)\left(U^{\prime} \Sigma_{w}{ }^{-1} U^{\prime T}\right)\left(U^{\prime} \Sigma_{w}{ }^{-1} U^{\prime T}\right.$

$\left.+\alpha^{2} V \Sigma^{-2} V^{T}\right)^{-1} V \Sigma^{-1} U^{T} \|$

$=\square\left(U \Sigma V^{T}\right)\left(V \Sigma^{-1} U^{T}\right)-\left(U \Sigma V^{T}\right)\left(U^{\prime} \Sigma_{w}{ }^{-1} U^{\prime T}\right)\left(U^{\prime} \Sigma_{w}{ }^{-1} U^{\prime T}\right.$

$\left.+\alpha^{2} V \Sigma^{-2} V^{T}\right)^{-1} V \Sigma^{-1} U^{T} \|$

$=\square\left(U \Sigma V^{T}\right)\left(I-U^{\prime} \Sigma_{w}{ }^{-1} U^{\prime T}\left(U^{\prime} \Sigma_{w}{ }^{-1} U^{\prime T}\right.\right.$

$\left.\left.+\alpha^{2} V \Sigma^{-2} V^{T}\right)^{-1}\right)\left(V \Sigma^{-1} U^{T}\right) \|$

Let $A \square U^{\prime} \Sigma_{w}^{-1} U^{\prime T} ; B \square V \Sigma^{-2} V^{T} ; C \square\left(V \Sigma^{-1} U^{T}\right) ; D \square\left(U \Sigma V^{T}\right)=J$

$E \square I-U^{\prime} \Sigma_{w}^{-1} U^{\prime T}\left(U^{\prime} \Sigma_{w}^{-1} U^{\prime T}+\alpha^{2} V \Sigma^{-2} V^{T}\right)^{-1}=I-A\left(A+\alpha^{2} B\right)^{-1}$

$\|D\|=\bar{\sigma}(J)=\bar{\sigma} ; \quad\|A\|=1 / \underline{\sigma}(W)=\underline{\sigma}_{w} ;\|C\|=1 / \underline{\sigma} ;\|B\|=1 / \underline{\sigma}^{2}$

$\square I+\Phi \square\|\| D\left(I-A A^{-1}\left(I+\alpha^{2} B A^{-1}\right)^{-1}\right) C \|$

$=\left\|D\left(I-\left(I+\alpha^{2} B A^{-1}\right)^{-1}\right) C\right\|=\left\|D\left(I+\alpha^{2} B A^{-1}\right)^{-1} \alpha^{2} B A^{-1} C\right\|$

$\square I+\Phi \square \leqslant\|D\|\left\|\left(I+\alpha^{2} B A^{-1}\right)^{-1}\right\|\left\|\alpha^{2} B A^{-1}\right\|\|C\|$

$\square I+\Phi \square \leq \frac{\|D\|\left\|\alpha^{2} B A^{-1}\right\|\|C\|}{\left\|\left(I+\alpha^{2} B A^{-1}\right)\right\|} \leq \frac{\|D\|\left\|\alpha^{2} B A^{-1}\right\|\|C\|}{1+\left\|\alpha^{2} B A^{-1}\right\|}$

$\left\|\alpha^{2} B A^{-1}\right\| \leq\left|\alpha^{2}\right|\left(1 / \underline{\sigma}^{2}\right) \bar{\sigma}_{w} \leq \bar{\sigma}_{w} \alpha^{2} / \underline{\sigma}^{2}$

$\square I+\Phi \square \leq \frac{\bar{\sigma} \bar{\sigma}_{w} \alpha^{2} / \underline{\sigma}^{2} \underline{\sigma}}{1+\bar{\sigma}_{w} \alpha^{2} / \underline{\sigma}^{2}} \leq\left(\frac{\bar{\sigma}}{\underline{\sigma}}\right) \cdot\left(\frac{\bar{\sigma}_{w} \alpha^{2}}{\underline{\sigma}^{2}+\bar{\sigma}_{w} \alpha^{2}}\right)$

$\leq \kappa \cdot\left(\frac{\bar{\sigma}_{w} \alpha^{2}}{\underline{\sigma}^{2}+\bar{\sigma}_{w} \alpha^{2}}\right) \approx \frac{\bar{\sigma}_{w} \alpha^{2}}{\underline{\sigma}^{2}+\bar{\sigma}_{w} \alpha^{2}}$

Although $\bar{\sigma}_{w}$ has no effect in $\left\|J_{w}^{\dagger}\right\|$ or $\left\|J^{h}\right\|$, but it has numerically validated using random matrices that numerical value of $\square I+\Phi \square$ has close approximation to $\left(\frac{\bar{\sigma}_{w} \alpha^{2}}{\underline{\sigma}^{2}+\bar{\sigma}_{w} \alpha^{2}}\right) ; \forall \alpha>0$ for wide range values for $W_{1}$. The condition number $\kappa$ steeply scales it during singularity crossings making $\beta^{\prime}$ very high which directly violates the stability conditions.

\section{APPENDIX- I.D}

$$
\begin{aligned}
& \forall G \square\left[\begin{array}{cc}
\alpha_{p} & \alpha_{p d} / 2 \\
\alpha_{p d} / 2 & \alpha_{d}
\end{array}\right] \\
& \Rightarrow \operatorname{det}(G-\lambda I)=\lambda^{2}-\lambda\left(\alpha_{p}+\alpha_{d}\right)+\alpha_{p} \alpha_{d}-\alpha_{p d}^{2} / 4 ;
\end{aligned}
$$

$\forall\left(\lambda_{1}^{\prime}, \lambda_{2}^{\prime}\right) \square$ roots of $\operatorname{det}(G-\lambda I)=0$

$\max \left(\lambda_{1}^{\prime}, \lambda_{2}^{\prime}\right)=\frac{\left(\alpha_{p}+\alpha_{d}\right)+\sqrt{\alpha_{p}^{2}+\alpha_{d}^{2}-2 \alpha_{p} \alpha_{d}+\alpha_{p d}^{2}}}{2}=\max (\operatorname{eigen}(G))$

$\because G$ is symmetric positive definite

$\|G\|=\max (\operatorname{eigen}(G))=\lambda_{1}$

$\|G\|=\frac{\left(\alpha_{p}+\alpha_{d}\right)+\sqrt{\left.\left(\alpha_{p}-\alpha_{d}\right)^{2}+\alpha_{p d}^{2}\right)}}{2 \sqrt{1+\rho^{2}}}$ 